\title{
HEISENBERG UNIQUENESS PAIRS FOR THE FOURIER TRANSFORM ON THE HEISENBERG GROUP
}

\author{
SOMNATH GHOSH AND R.K. SRIVASTAVA
}

\begin{abstract}
In this article, we prove that (unit sphere, non-harmonic cone) is a Heisenberg uniqueness pair for the symplectic Fourier transform on $\mathbb{C}^{n}$. We derive that spheres as well as non-harmonic cones are determining sets for the spectral projections of the finite measure supported on the unit sphere. Further, we prove that if the Fourier transform of a finitely supported function on step two nilpotent Lie group is of arbitrary finite rank, then the function must be zero. The latter result correlates to the annihilating pair for the Weyl transform.
\end{abstract}

\section{INTRODUCTION}

Let $\Gamma$ be a finite disjoint union of smooth curves in the plane $\mathbb{R}^{2}$. Let $X(\Gamma)$ be the space of all finite complex-valued Borel measures $\mu$ in $\mathbb{R}^{2}$, which are supported on $\Gamma$ and absolutely continuous with respect to the arc length measure of $\Gamma$. For $(\xi, \eta) \in \mathbb{R}^{2}$, the Fourier transform of $\mu$ can be defined by

$$
\hat{\mu}(\xi, \eta)=\int_{\Gamma} e^{-i(x \cdot \xi+y \cdot \eta)} d \mu(x, y) .
$$

In the above context, $\hat{\mu}$ becomes uniformly continuous bounded function. Thus, we can analyze the pointwise vanishing property of $\hat{\mu}$.

Definition 1.1. Let $\Lambda$ be a set in $\mathbb{R}^{2}$. The pair $(\Gamma, \Lambda)$ is called a Heisenberg uniqueness pair for $X(\Gamma)$ if the only $\mu \in X(\Gamma)$ that satisfies $\left.\hat{\mu}\right|_{\Lambda}=0$ is $\mu=0$.

In general, the problem of Heisenberg uniqueness pair (HUP) is a question about the determining property of the finite Borel measures, which are supported on some given lower dimensional entities whose Fourier transform also vanishes on lower dimensional entities. In particular, if $\Gamma$ is compact, then by Paley Wiener theorem $\hat{\mu}$ is real analytic having exponential growth, and hence $\hat{\mu}$ can vanish only on a set of measure zero. Thus, the HUP problem becomes a little easier in this case. However, this problem becomes immensely difficult while the measure is supported on a non-compact entity. Further, it appears that the problem of HUP is a natural variant of the uncertainty principle for the Fourier transform.

Date: August 6, 2020.

2000 Mathematics Subject Classification. Primary 42A38; Secondary 44A35.

Key words and phrases. Convolution, Fourier transform, Heisenberg group, Laguerre polynomial. 
In addition, the concept of determining the Heisenberg uniqueness pair has a correlation with the concept of annihilating pair. Let $A \subseteq \mathbb{R}$ and $\Sigma \subseteq \hat{\mathbb{R}}$ be measurable subsets. Then the pair $(A, \Sigma)$ is called a weak annihilating pair (WAP) if $\operatorname{supp} f \subseteq A$ and $\operatorname{supp} \hat{f} \subseteq \Sigma$, implies $f=0$. The pair $(A, \Sigma)$ is called a strong annihilating pair (SAP) if there exists a positive number $C=C(A, \Sigma)$ such that

$$
\|f\|_{2}^{2} \leq C\left(\|f\|_{L^{2}\left(A^{c}\right)}+\|\hat{f}\|_{L^{2}\left(\Sigma^{c}\right)}\right)
$$

for all $f \in L^{2}(\mathbb{R})$. It is obvious from (1.1) that every SAP is a WAP. In [6], Benedicks had, eventually, proved that $(A, \Sigma)$ is a WAP if $A$ and $\Sigma$ both have finite measure, whereas it follows from Amrein-Berthier [2] that $(A, \Sigma)$ is a $\mathrm{SAP}$ under the identical assumption as in [6]. For the latter conclusion we refer to [20. Later, various analogues of Benedicks-Amrein-Berthier theorem and qualitative uncertainty principle, have been investigated in different setups (see [7, 15, 20, 24, 27, 31, 34]).

In [27], Narayanan and Ratnakumar proved that if $f \in L^{1}\left(\mathbb{H}^{n}\right)$ is supported on $B \times \mathbb{R}$, where $B$ is a compact subset of $\mathbb{C}^{n}$, and if $\hat{f}(\lambda)$ is of finite rank for each $\lambda$, then $f=0$. Thereafter, Vemuri [44 replaced the compactness condition on $B$ by finite measure. In [10, authors considered $B$ as a rectangle in $\mathbb{R}^{2 n}$ to prove analogous result in step two nilpotent Lie group.

In this article, we prove the result on the general step two nilpotent Lie group when $B$ is an arbitrary set of finite measure, using the Hilbert space theory. Though specifying the appropriate set of projections in the general setups was a major bottleneck, and we sorted out the same. For the sake of simplicity, we prefer to prove the result for the case of the Heisenberg group as the same technique can be extended for the general step two nilpotent Lie group. Finally, we consider the case of SAP for the Weyl transform and draw some conclusions therein.

We discuss the concept of HUP, which was introduced by Hedenmalm and Montes-Rodríguez in [21, and then inn [21], they have shown that the pair (hyperbola, some discrete set) is a Heisenberg uniqueness pair. As a dual problem, a weak* dense subspace of $L^{\infty}(\mathbb{R})$ has been constructed to solve the long standing Klein-Gordon equation. Further, Hedenmalm and Montes-Rodríguez 21] have given a complete characterization of the Heisenberg uniqueness pairs corresponding to any two parallel lines.

Lev [26] and Sjölin [35] have independently shown that circle and certain system of lines form HUP corresponding to the unit circle. Further, Vieli [45] has generalized the case of circle in the higher dimension and shows that a sphere whose radius does not lie in the zero sets of the Bessel functions $J_{(n+2 k-2) / 2} ; k \in \mathbb{Z}_{+}$, the set of non-negative integers, is a HUP corresponding to the unit sphere $S^{n-1}$. In [40, the author has shown that a cone is a HUP corresponding to the sphere as long as the cone does not completely recline on the level surface of any homogeneous harmonic polynomial on $\mathbb{R}^{n}$. 
Further, Sjölin [36] derived some Heisenberg uniqueness pairs corresponding to the parabola. It has been extended to the case of paraboloid by Vieli [46]. Subsequently, Babot [5] has given a characterization of the Heisenberg uniqueness pairs corresponding to a certain system of three parallel lines. Thereafter, the authors in [17] have given some necessary and sufficient conditions for the Heisenberg uniqueness pairs corresponding to a system of four parallel lines. However, the question of the unique necessary and sufficient condition for the finitely many parallel lines as compared to three lines result [5] is still unsolved. For more examples of HUP corresponding to spiral, exponential curves, sphere and paraboloid, see [9, 17].

Jaming and Kellay [25] have given a unifying proof for some of the Heisenberg uniqueness pairs corresponding to the hyperbola, polygon, ellipse and graph of the functions $\varphi(t)=|t|^{\alpha}$, whenever $\alpha>0$. Thereafter, Gröchenig and Jaming [19] have worked out some of the Heisenberg uniqueness pairs corresponding to quadratic surfaces. For more results on HUP via ergodic theory dynamical systems, see [8, 22, 23, 16].

In this article, we work out a few analogous results on the Heisenberg group in various aspects. Firstly, we consider the symplectic Fourier transform (SFT) on $\mathbb{C}^{n}$. We prove that $S^{2 n-1}$ forms a HUP with a non-harmonic complex cone for SFT. The above result has a sharp contrast with analogous result for the Euclidean Fourier transform (EFT) on $\mathbb{R}^{2 n}$. Since a non-trivial complex cone in $\mathbb{C}^{n}(n \geq 2)$ can have topological dimension at most $2 n-2$, it follows that a $(2 n-2)$ - dimensional entity forms a HUP with $S^{2 n-1}$ for SFT. Although for $\mathrm{EFT}$ on $\mathbb{R}^{2 n}$, the least topological dimension required (in general) for a set to be a HUP with unit sphere $S^{2 n-1}$ is $2 n-1$. We also observe that the conclusion of the above result for the SFT holds true for a real non-harmonic cone in $\mathbb{R}^{2 n}$.

Thereafter, we consider the case of modified Fourier transform on the Heisenberg group. We prove that a finite measure supported on the cylinder $S^{2 n-1} \times \mathbb{R}$ can be determined by a non-harmonic cone as well as the boundary of a bounded domain in $\mathbb{C}^{n}$.

Further, we consider reasonably more interesting case of determining a finite measure $\mu$ which is supported on $S^{2 n-1}$, in terms of its spectral projections. We prove that if the spectral projections $\varphi_{k}^{n-1} \times \mu$ vanish on a sphere of arbitrary radius, then $\mu$ is trivial. We observed that the above measures can also be determined by a non-harmonic complex cone. Though, the case of the real non-harmonic cone is yet to be settled, we leave it open for the time being.

\section{Preliminaries}

In this section, we describe some basic facts about Fourier transform on the Heisenberg group, Weyl transform, special Hermite functions and expansion of functions on $\mathbb{C}^{n}$ accordingly. Subsequently, we mention some auxiliary results 
for the bigraded spherical harmonics and non-harmonic real as well as complex cone.

The Heisenberg group $\mathbb{H}^{n}=\mathbb{C}^{n} \times \mathbb{R}$ is a step two nilpotent Lie group having center $\mathbb{R}$ that equipped with the group law

$$
(z, t) \cdot(w, s)=\left(z+w, t+s+\frac{1}{2} \operatorname{Im}(z \cdot \bar{w})\right) .
$$

By Stone-von Neumann theorem, the infinite dimensional irreducible unitary representations of $\mathbb{H}^{n}$ can be parameterized by $\mathbb{R}^{*}=\mathbb{R} \backslash\{0\}$. That is, each of $\lambda \in \mathbb{R}^{*}$ defines a Schrödinger representation $\pi_{\lambda}$ of $\mathbb{H}^{n}$ by

$$
\pi_{\lambda}(z, t) \varphi(\xi)=e^{i \lambda t} e^{i \lambda\left(x \cdot \xi+\frac{1}{2} x \cdot y\right)} \varphi(\xi+y),
$$

where $z=x+i y$ and $\varphi \in L^{2}\left(\mathbb{R}^{n}\right)$. Hence, the group Fourier transform of $f \in L^{1}\left(\mathbb{H}^{n}\right)$ can be defined by

$$
\hat{f}(\lambda)=\int_{\mathbb{H}^{n}} f(z, t) \pi_{\lambda}(z, t) d z d t
$$

is a bounded operator. When $f \in L^{2}\left(\mathbb{H}^{n}\right), \hat{f}(\lambda)$ is a Hilbert-Schmidt operator. An important technique in many problems on $\mathbb{H}^{n}$ is to take partial Fourier transform in the $t$-variable to reduce the matter to $\mathbb{C}^{n}$. Let

$$
f^{\lambda}(z)=\int_{\mathbb{R}} f(z, t) e^{i \lambda t} d t
$$

be the inverse Fourier transform of $f$ in the $t$-variable. The group convolution of the functions $f, g \in L^{1}\left(\mathbb{H}^{n}\right)$, defined by

$$
f * g(z, t)=\int_{H^{n}} f((z, t)(-w,-s)) g(w, s) d w d s,
$$

by taking the inverse Fourier transform in the $t$-variable, takes the form

$$
\begin{aligned}
(f * g)^{\lambda}(z) & =\int_{-\infty}^{\infty} f * g(z, t) e^{i \lambda t} d t \\
& =\int_{\mathbb{C}^{n}} f^{\lambda}(z-w) g^{\lambda}(w) e^{\frac{i \lambda}{2} \operatorname{Im}(z \cdot \bar{w})} d w \\
& =f^{\lambda} \times_{\lambda} g^{\lambda}(z),
\end{aligned}
$$

where $f^{\lambda} \times_{\lambda} g^{\lambda}$ is known as $\lambda$-twisted convolution. Thus, the group convolution $f * g$ on the Heisenberg group can be studied by the $\lambda$-twisted convolution $f^{\lambda} \times_{\lambda} g^{\lambda}$ on $\mathbb{C}^{n}$. When $\lambda \neq 0$, by a scaling argument, it is enough to study the twisted convolution for the case $\lambda=1$.

Now, we recall the Weyl transform, which is the most non-commutative constituent of the group Fourier transform on the Heisenberg group. Denote 
by $\pi_{\lambda}(z)=\pi_{\lambda}(z, 0)$. Then $\pi_{\lambda}(z, t)=e^{i \lambda t} \pi_{\lambda}(z)$. For a suitable function $g$ on $\mathbb{C}^{n}$, the Weyl transform of $g$ can be expressed by

$$
W_{\lambda}(g)=\int_{\mathbb{C}^{n}} g(w) \pi_{\lambda}(w) d w .
$$

This, in turn, implies $\hat{f}(\lambda)=W_{\lambda}\left(f^{\lambda}\right)$. It is easy followed that $W_{\lambda}(g)$ is a bounded operator, whenever $g \in L^{1}\left(\mathbb{C}^{n}\right)$. On the other hand, if $g \in L^{2}\left(\mathbb{C}^{n}\right)$, $W_{\lambda}(g)$ is a Hilbert-Schmidt opertor and satisfies the Plancherel formula

$$
|\lambda|^{\frac{n}{2}}\left\|W_{\lambda}(g)\right\|_{H S}=(2 \pi)^{\frac{n}{2}}\|g\|_{2}
$$

The Fourier-Winger transform of $\varphi, \psi \in L^{2}\left(\mathbb{R}^{n}\right)$ is defined by the formula $V_{\varphi}^{\psi}(z)=(2 \pi)^{-n / 2}\langle\pi(z) \varphi, \psi\rangle$. It is known that $V_{\varphi}^{\psi} \in L^{2}\left(\mathbb{C}^{n}\right)$ and satisfies the identity

$$
\int_{\mathbb{C}^{n}} V_{\varphi_{1}}^{\psi_{1}}(z) \overline{V_{\varphi_{2}}^{\psi_{2}}(z)} d z=\left\langle\varphi_{1}, \varphi_{2}\right\rangle \overline{\left\langle\psi_{1}, \psi_{2}\right\rangle},
$$

whenever $\varphi_{l}, \psi_{l} \in L^{2}\left(\mathbb{R}^{n}\right) ; l=1,2$. See [42].

Next, we describe the special Hermite expansion for function on $\mathbb{C}^{n}$. Let

$$
T=\frac{\partial}{\partial t}, X_{j}=\frac{\partial}{\partial x_{j}}+\frac{1}{2} y_{j} \frac{\partial}{\partial t} \text { and } Y_{j}=\frac{\partial}{\partial y_{j}}-\frac{1}{2} x_{j} \frac{\partial}{\partial t}
$$

be the left-invariant vector fields on $\mathbb{H}^{n}$. Then $\left\{T, X_{j}, Y_{j}: j=1, \ldots, n\right\}$ forms a basis for the Lie algebra $\mathfrak{h}^{n}$ of $\mathbb{H}^{n}$, and the representation $\pi_{\lambda}$ induces a representation $\pi_{\lambda}^{*}$ of $\mathfrak{h}^{n}$ on the space of $C^{\infty}$ vectors in $L^{2}\left(\mathbb{R}^{n}\right)$ via

$$
\pi_{\lambda}^{*}(X) f=\left.\frac{d}{d t}\right|_{t=0} \pi_{\lambda}(\exp t X) f .
$$

It is easy to see that $\pi_{\lambda}^{*}\left(X_{j}\right)=i \lambda x_{j}$ and $\pi_{\lambda}^{*}\left(Y_{j}\right)=\frac{\partial}{\partial x_{j}}$. Hence for the subLaplacian $\mathcal{L}=-\sum_{j=1}^{n}\left(X_{j}^{2}+Y_{j}^{2}\right)$, it follows that $\pi_{\lambda}^{*}(\mathcal{L})=-\Delta_{x}+\lambda^{2}|x|^{2}=: H_{\lambda}$, the scaled Hermite operators. Let $\phi_{\alpha}^{\lambda}(x)=|\lambda|^{\frac{n}{4}} \phi_{\alpha}(\sqrt{|\lambda|} x) ; \alpha \in \mathbb{Z}_{+}^{n}$, where $\phi_{\alpha}$ 's are the Hermite functions on $\mathbb{R}^{n}$. Then each $\phi_{\alpha}^{\lambda}$ is an eigenfunction of $H_{\lambda}$ with eigenvalue $(2|\alpha|+n)|\lambda|$. Hence the entry functions $E_{\alpha \beta}^{\lambda}$ 's of the representation $\pi_{\lambda}$ are eigenfunctions of the sub-Laplacian $\mathcal{L}$ satisfying

$$
\mathcal{L} E_{\alpha \beta}^{\lambda}=(2|\alpha|+n)|\lambda| E_{\alpha \beta}^{\lambda},
$$

where $E_{\alpha \beta}^{\lambda}(z, t)=\left\langle\pi_{\lambda}(z, t) \phi_{\alpha}^{\lambda}, \phi_{\beta}^{\lambda}\right\rangle$. Since $E_{\alpha \beta}^{\lambda}(z, t)=e^{i \lambda t}\left\langle\pi_{\lambda}(z) \phi_{\alpha}^{\lambda}, \phi_{\beta}^{\lambda}\right\rangle$, the eigenfunctions $E_{\alpha \beta}^{\lambda}$ 's are not in $L^{2}\left(\mathbb{H}^{n}\right)$. However, for each fixed $t$, they are in $L^{2}\left(\mathbb{C}^{n}\right)$. Now, define an operator $L_{\lambda}$ by $\mathcal{L}\left(e^{i \lambda t} f(z)\right)=e^{i \lambda t} L_{\lambda} f(z)$. Then the special Hermite function

$$
\phi_{\alpha \beta}^{\lambda}(z)=(2 \pi)^{-\frac{n}{2}}\left\langle\pi_{\lambda}(z) \phi_{\alpha}^{\lambda}, \phi_{\beta}^{\lambda}\right\rangle
$$

is an eigenfunction of $L_{\lambda}$ with eigenvalue $2|\alpha|+n$. Now, we can summarize that the special Hermite functions $\phi_{\alpha \beta}^{\lambda}$ 's form an orthonormal basis for $L^{2}\left(\mathbb{C}^{n}\right)$ 
[43]. Hence $g \in L^{2}\left(\mathbb{C}^{n}\right)$ can be expressed as

$$
g=\sum_{\alpha, \beta}\left\langle g, \phi_{\alpha \beta}^{\lambda}\right\rangle \phi_{\alpha \beta}^{\lambda} .
$$

By employing a correlation of the special Hermite functions with the Laguerre function, expression (2.3) can be further simplified, for which we need to recall the definition of Laguerre function. Given $v \in \mathbb{C}$, the Laguerre polynomial of degree $k \in \mathbb{Z}_{+}$is defined by

$$
L_{k}^{v}(x)=\sum_{j=0}^{k}\left(\begin{array}{l}
v+k \\
k-j
\end{array}\right) \frac{(-x)^{j}}{j !} .
$$

Now, Laguerre function on $\mathbb{C}^{n}$ of order $n-1$ and degree $k$ can be defined by $\varphi_{k}^{n-1}(z)=L_{k}^{n-1}\left(\frac{|z|^{2}}{2}\right) e^{-\frac{|z|^{2}}{4}}$. Denote $\varphi_{k, \lambda}^{n-1}(z)=\varphi_{k}^{n-1}(\sqrt{|\lambda|} z)$, where $\lambda \in \mathbb{R}^{*}$. Then the special Hermite functions $\phi_{\alpha \alpha}^{\lambda}$ will satisfy the relation

$$
\sum_{|\alpha|=k} \phi_{\alpha, \alpha}^{\lambda}(z)=(2 \pi)^{-\frac{n}{2}}|\lambda|^{\frac{n}{2}} \varphi_{k, \lambda}^{n-1}(z)
$$

Thus, $g \in L^{2}\left(\mathbb{C}^{n}\right)$ can be expressed by

$$
g(z)=(2 \pi)^{-n}|\lambda|^{n} \sum_{k=0}^{\infty} g \times_{\lambda} \varphi_{k, \lambda}^{n-1}(z) .
$$

Please refer to [43. In particular, for $\lambda=1$, we have

$$
g(z)=(2 \pi)^{-n} \sum_{k=0}^{\infty} g \times \varphi_{k}^{n-1}(z),
$$

which is the special Hermite expansion of $g$. Hence $g$ can be completely determined by its spectral projections $g \times \varphi_{k}^{n-1}$. Thus, it is an interesting question to determine finite measures $\mu$ those are supported on a thin set in $\mathbb{C}^{n}$ via spectral projections $\varphi_{k}^{n-1} \times \mu$. We discuss this assertion in Section 5 .

Let $P_{p, q}$ denote the space of all bi-graded homogeneous polynomials on $\mathbb{C}^{n}$ of the form

$$
P(z)=\sum_{|\alpha|=p} \sum_{|\beta|=q} c_{\alpha \beta} z^{\alpha} \bar{z}^{\beta}
$$

where $p, q \in \mathbb{Z}_{+}$. Denote $H_{p, q}=\left\{P \in P_{p, q}: \Delta P=0\right\}$. The restriction of bigraded homogeneous harmonic polynomial to the unit sphere $S^{2 n-1}$ is called bi-graded spherical harmonic.

The following weighted functional relations can be obtained by considering the Hecke-Bochner identity for the spectral projection of compactly supported functions. For more details, see [43], p. 98. 
Lemma 2.1. [43] For $z \in \mathbb{C}^{n}$, let $P \in H_{p, q}$ and $d \nu_{r}=P d \sigma_{r}$, where $\sigma_{r}$ is the surface measure on the sphere $S_{r}$. Then

$$
\varphi_{k}^{n-1} \times \nu_{r}(z)=(2 \pi)^{-n} \frac{\Gamma(k-q+1)}{\Gamma(k+n+p)} r^{2(p+q)} \varphi_{k-q}^{n+p+q-1}(r) P(z) \varphi_{k-q}^{n+p+q-1}(z),
$$

if $k \geq q$ and 0 otherwise.

We need the following basic facts about the bigraded spherical harmonics, (see [12, 18, 43] for details). Let $K=U(n)$ be the unitary group and $M=$ $U(n-1)$. Then, $S^{2 n-1} \cong K / M$ under the map $k M \rightarrow k . e_{n}$, where $k \in U(n)$ and $e_{n}=(0, \ldots, 1) \in \mathbb{C}^{n}$. Let $\hat{K}_{M}$ denote the set of all equivalence classes of irreducible unitary representations of $K$ which have a nonzero $M$-fixed vector.

For a $\tau \in \hat{K}_{M}$, which is realized on $V_{\tau}$, let $\left\{e_{1}, \ldots, e_{d(\tau)}\right\}$ be an orthonormal basis of $V_{\tau}$ with $e_{1}$ as the $M$-fixed vector. Let $t_{i j}^{\tau}(k)=\left\langle e_{i}, \tau(k) e_{j}\right\rangle, k \in K$. By the Peter-Weyl theorem, $\left\{\sqrt{d(\tau)} t_{j 1}^{\tau}: 1 \leq j \leq d(\tau), \tau \in \hat{K}_{M}\right\}$ forms an orthonormal basis for $L^{2}(K / M)$, (see [43]). Define $Y_{j}^{\tau}(\omega)=\sqrt{d(\tau)} t_{j 1}^{\tau}(k)$, where $\omega=k \cdot e_{n} \in S^{2 n-1}$, and $k \in K$. Then $\left\{Y_{j}^{\tau}: 1 \leq j \leq d(\tau), \tau \in \hat{K}_{M}\right\}$ becomes an orthonormal basis for $L^{2}\left(S^{2 n-1}\right)$.

Since $H_{p, q}$ is $K$-invariant, let $\pi_{p, q}$ be the corresponding representation of $K$ on $H_{p, q}$. Then $\hat{K}_{M}$ can be identified with $\left\{\pi_{p, q}: p, q \in \mathbb{Z}_{+}\right\}$. See [33], p.253, for more details. Thus, a bi-graded spherical harmonic on $S^{2 n-1}$ can be defined by $Y_{j}^{p, q}(\omega)=\sqrt{d(p, q)} t_{j 1}^{p, q}(k)$, and hence $\left\{Y_{j}^{p, q}: 1 \leq j \leq d(p, q), p, q \in \mathbb{Z}_{+}\right\}$ forms an orthonormal basis for $L^{2}\left(S^{2 n-1}\right)$. For $f \in L^{2}\left(S^{2 n-1}\right)$, the expression

$$
\Pi_{p, q}(f)(\omega)=\sum_{j=1}^{d(p, q)} a_{j}^{p, q} Y_{j}^{p, q}(\omega),
$$

where $a_{j}^{p, q} \in \mathbb{C}$, is called $(p, q)^{t h}$ spherical harmonic projection of $f$.

For each $l \in \mathbb{Z}_{+}$, the space $H_{l}$ consists of spherical harmonics of degree $l$ in $\mathbb{R}^{d}$, is $S O(d)$ - invariant. When $d=2 n, H_{l}$ will be $U(n)$ - invariant as well, and under this action of $U(n)$, the space $H_{l}$ breaks up into an orthogonal direct sum of $H_{p, q}$ 's, where $p+q=l$.

Lemma 2.2. [33]. Let $\omega \in S^{2 n-1}$ and $Y \in H_{l}$. Then there exists $Y_{p, q} \in$ $H_{p, q}, p+q=l$ such that

$$
Y(\omega)=\sum_{p+q=l} Y_{p, q}(\omega)
$$

Definition 2.3. A set $\mathcal{C} \subset \mathbb{C}^{n}(n \geq 2)$ that satisfies the scaling condition $\lambda \mathcal{C} \subseteq \mathcal{C}$ for all $\lambda \in \mathbb{C}$, is called a complex cone, whereas a set $\mathcal{D}$ in $\mathbb{R}^{d}(d \geq 2)$ which satisfies $\lambda \mathcal{D} \subseteq \mathcal{D}$, for all $\lambda \in \mathbb{R}$ is called a real cone.

We say a cone is non-harmonic if it is not contained in the zero set of any homogeneous harmonic polynomial. An example of a non-harmonic complex cone was produced by the author (see [39]). The zero set of the polynomial 
$H(z)=a z_{1} \bar{z}_{2}+|z|^{2}$, where $a \neq 0$ and $z \in \mathbb{C}^{n}$, is a complex cone which is not contained in the zero set of any bi-graded homogeneous harmonic polynomial.

An example of a non-harmonic real cone was given by Armitage, (see [4]). Let $0<a<1$. Then $K_{a}=\left\{x \in \mathbb{R}^{d}:\left|x_{1}\right|^{2}=a^{2}|x|^{2}\right\}$ is a non-harmonic cone if and only if $D^{m} G_{k}^{\frac{d-2}{2}}(a) \neq 0$, for all $0 \leq m \leq k-2$, where $D^{m}$ stands for the $m$ th derivative on $\mathbb{R}$.

In view of Lemma 2.2, it is easy to prove the following result, which is required to prove our main result.

Lemma 2.4. Let $Y \in H_{l}$ be given as in (2.8). Suppose $\mathcal{C}$ be a complex cone, and denote $\widetilde{\mathcal{C}}=\left\{\frac{z}{|z|}: z \in \mathcal{C}, z \neq 0\right\}$. Then $Y=0$ on $\widetilde{\mathcal{C}}$ if and only if $Y_{p, q}=0$ on $\widetilde{\mathcal{C}}, \forall p, q \in \mathbb{Z}_{+}$which are lying on the diagonal $p+q=l$.

Proof. Let $\omega \in \widetilde{\mathcal{C}}$ and $Y(\omega)=0$. Since the cone $\mathcal{C}$ is closed under complex scaling, by replacing $\omega$ with $e^{i \theta} \omega$ in (2.8) we get

$$
\sum_{p+q=l} e^{i(p-q) \theta} Y_{p, q}(\omega)=0
$$

Thus, the proof of the required lemma will be followed by the fact that $\left\{e^{i s \theta}: s \in \mathbb{Z}\right\}$ is an orthogonal set in $L^{2}\left(S^{1}\right)$.

For each fixed $\xi \in S^{2 n-1}$, define a linear functional on $H_{l}$ by $Y \mapsto Y(\xi)$. Then there exists a unique spherical harmonic, say $Z_{\xi}^{(l)} \in H_{l}$ such that

$$
Y(\xi)=\int_{S^{d-1}} Z_{\xi}^{(l)}(\eta) Y(\eta) d \sigma(\eta)
$$

The spherical harmonic $Z_{\xi}^{(l)}$ is a $S O(2 n)$ bi-invariant real-valued function, which is constant on each geodesic, orthogonal to the line joining the origin and $\xi$. The spherical harmonic $Z_{\xi}^{(l)}$ is called the zonal harmonic of the space $H_{l}$ at the pole $\xi$. For more details, see [41], p. 143.

For $1 \leq t \leq \infty$, let $f \in L^{t}\left(S^{2 n-1}\right)$. For each $l \in \mathbb{Z}_{+}$, we define the $l^{t h}$ spherical harmonic projection of $f$ by

$$
\Pi_{l} f(\xi)=\int_{S^{2 n-1}} Z_{\xi}^{(l)}(\eta) f(\eta) d \sigma(\eta) .
$$

Then $\Pi_{l} f$ is a spherical harmonic of degree $l$. If for a $\delta>n-1$, we denote $A_{l}^{m}(\delta)=\left(\begin{array}{c}m-l+\delta \\ \delta\end{array}\right)\left(\begin{array}{c}m+\delta \\ \delta\end{array}\right)^{-1}$, then the spherical harmonic expansion $\sum_{l=0}^{\infty} \Pi_{l} f$ will be $\delta$-Cesaro summable to $f$. That is,

$$
f=\lim _{m \rightarrow \infty} \sum_{l=0}^{m} A_{l}^{m}(\delta) \Pi_{l} f,
$$


where limit in the right-hand side of (2.11) exists in $L^{t}\left(S^{2 n-1}\right)$. Further, the convergence in (2.11) can be extended to hold in $L^{t}\left(r S^{2 n-1}\right)$ while $r>0$. For more details, see [37].

We would like to mention that the proof of our main result will be carried out by concentrating the cone to the unit sphere and decomposing the integral on sphere into averages over geodesic spheres. This is possible because the cone is closed under scaling.

For $\omega \in S^{2 n-1}$ and $t \in(-1,1)$, the set $S_{\omega}^{t}=\left\{\nu \in S^{2 n-1}: \omega \cdot \nu=t\right\}$ is a geodesic sphere on $S^{2 n-1}$ with a pole at $\omega$. Let $f$ be an integrable function on $S^{2 n-1}$. Then in view of Fubini's Theorem, we can define the geodesic spherical means of $f$ by

$$
\tilde{f}(\omega, t)=\int_{S_{\omega}^{t}} f d \sigma_{2 n-2}
$$

where $\sigma_{2 n-2}$ stands for the normalized surface measure on the geodesic sphere $S_{\omega}^{t}$.

Since the zonal harmonic $Z_{\xi}^{(l)}$ is $S O(2 n)$ bi-invariant, three exists a nice function $F$ on $(-1,1)$ satisfying $Z_{\xi}^{(l)}(\eta)=F(\xi \cdot \eta)$. Hence the extension of the formula (2.9) for the functions $F \in L^{1}(-1,1)$ is inevitable. The latter fact is known as Funk-Hecke formula. In other words,

$$
\int_{S^{2 n-1}} F(\xi \cdot \eta) Y(\eta) d \sigma(\eta)=C_{l} Y(\xi)
$$

where the constant $C_{l}$ is given by

$$
C_{l}=\alpha_{l} \int_{-1}^{1} F(t) G_{l}^{n-1}(t)\left(1-t^{2}\right)^{\frac{2 n-3}{2}} d t
$$

and $G_{l}^{\beta}$ stands for the Gegenbauer polynomial of degree $l$ and order $\beta$. For more details, see [3], p. 459. We shall mention the following lemma which percolates the geodesic mean vanishing conditions of $f \in L^{1}\left(S^{2 n-1}\right)$ to vanishing condition of each spherical harmonic projection of $f$. For the class of continuous functions $C\left(S^{2 n-1}\right)$, this lemma was proved in [1]. In [40, the author has extended the result for functions in $L^{1}\left(S^{2 n-1}\right)$ with the help of the Cesaro summation formula (2.11).

Lemma 2.5. [40] Let $f \in L^{1}\left(S^{2 n-1}\right)$. Then $\tilde{f}(\omega, t)=0$ for all $t \in(-1,1)$ if and only if $\Pi_{l} f(\omega)=0$ for all $l \in \mathbb{Z}_{+}$.

Notice that, as a corollary to Lemma 2.5, it can be shown that if $\tilde{f}(\omega, t)=0$ for all $(\omega, t) \in \widetilde{\mathcal{C}} \times(-1,1)$, then $f=0$ on $S^{2 n-1}$ as long as $\mathcal{C}$ is happened to be non-harmonic. 


\section{UNIQUENESS PAIRS FOR THE SYMPLECTIC FOURIER TRANSFORM}

In this section, we prove that the unit sphere $S^{2 n-1}$ together with a nonharmonic complex cone forms a Heisenberg uniqueness pair for the symplectic Fourier transform (SFT).

Let $X\left(S^{2 n-1}\right)$ denote the space of all finite Borel measures $\mu$ in $\mathbb{C}^{n}$ which are supported on $S^{2 n-1}$ and absolutely continuous with respect to the surface measure on $S^{2 n-1}$. By Radon-Nikodym theorem, there exists $f \in L^{1}\left(S^{2 n-1}\right)$ such that $d \mu=f d \sigma$. Define the symplectic Fourier transform of a measure $\mu \in X\left(S^{2 n-1}\right)$ by

$$
\mathcal{F}_{S} \mu(z)=\int_{S^{2 n-1}} e^{-\frac{i}{2} \operatorname{Im}(z \cdot \bar{\zeta})} f(\zeta) d \sigma(\zeta),
$$

where $z=x+i y \in \mathbb{C}^{n}$ and $\zeta=\xi+i \eta \in \mathbb{C}^{n}$. Hence $\mathcal{F}_{S} \mu$ is a bounded uniformly continuous function on $\mathbb{C}^{n}$. In other words, $\mathcal{F}_{S} \mu$ can be expressed as

$$
\mathcal{F}_{S} \mu(x, y)=\int_{S^{2 n-1}} e^{-\frac{i}{2}(-x \cdot \eta+y \cdot \xi)} f(\xi, \eta) d \sigma(\xi, \eta) .
$$

We are intended to prove the following result.

Theorem 3.1. Let $\mathcal{C}$ be a complex cone in $\mathbb{C}^{n}$ and $\mu \in X\left(S^{2 n-1}\right)$. If $\mathcal{F}_{S} \mu(z)=$ 0 for all $z \in \mathcal{C}$, then $\mu=0$ if and only if $\mathcal{C}$ is non-harmonic.

Proof. Let $(x, y)=r \omega$, where $\omega=\left(\omega_{1}, \ldots, \omega_{n}, \omega_{1}^{\prime}, \ldots, \omega_{n}^{\prime}\right) \in S^{2 n-1}$. Denote $\tilde{\omega}=\left(\omega_{1}^{\prime}, \ldots, \omega_{n}^{\prime},-\omega_{1}, \ldots,-\omega_{n}\right)$. Then from (3.1) it implies that

$$
\int_{S^{2 n-1}} e^{-\frac{i}{2} r \tilde{\omega} \cdot(\xi, \eta)} f(\xi, \eta) d \sigma(\xi, \eta)=0
$$

whenever $r \omega \in \mathcal{C}$. Since $\mathcal{C}$ is closed under complex scaling, $r \omega \in \mathcal{C}$ implies $r \tilde{\omega} \in \mathcal{C}$. By decomposing the integral in (3.2) over geodesic spheres at pole $\omega$, we obtain

$$
\int_{-1}^{1}\left(\int_{S_{\omega}^{t}} e^{-\frac{i}{2} r \omega \cdot \nu} f(\nu) d \sigma_{2 n-2}(\nu)\right) d t=0,
$$

where $S_{\omega}^{t}=\left\{\nu \in S^{2 n-1}: \omega \cdot \nu=t\right\}$. That is,

$$
\int_{-1}^{1} e^{-\frac{i}{2} r t} \tilde{f}(\omega, t) d t=0
$$

for all $r>0$. Hence $\tilde{f}(\omega, t)=0$, for all $t \in(-1,1)$. Thus, by Lemma 2.5, it follows that $\Pi_{l}(f)(\omega)=0$ for all $l \in \mathbb{Z}_{+}$. Further, by Lemma 2.4, we get $\Pi_{p, q} f(\omega)=0$ for all $p, q \in \mathbb{Z}_{+}$. Thus, by the given condition that $w \notin Y_{p q}^{-1}(0)$ for any $p, q \in \mathbb{Z}_{+}$, it follows that $f=0$. That is, $\mu=0$.

Conversely, assume that $\mathcal{C}$ is contained in the zero set of a bigraded homogeneous harmonic polynomial $P \in H_{p, q}$ and denote $Y=\left.P\right|_{S^{2 n-1}}$. For $\zeta \in S^{2 n-1}$, let $d \mu(\zeta)=Y(\zeta) d \sigma(\zeta)$. Then $\mu$ is a finite complex Borel measure supported 
on $S^{2 n-1}$. By identifying $\mathbb{C}^{n}$ with $\mathbb{R}^{2 n}$, we find that $H_{p, q} \subseteq H_{p+q}$. Therefore, for $z=r \omega \in \mathcal{C}$, we can summarize that

$$
\mathcal{F}_{S} \mu(z)=\int_{S^{2 n-1}} e^{-i r \tilde{\omega} \cdot \zeta} Y_{p+q}(\zeta) d \sigma(\zeta)=(2 \pi)^{n} i^{p+q} \frac{J_{p+q+n-1}(r)}{r^{n-1}} Y_{p+q}(\tilde{\omega}),
$$

where the last identity was obtain in [3], as a corollary of the Funk-Hecke formula (2.12). Recall that $r \omega \in \mathcal{C}$ implies $r \tilde{\omega} \in \mathcal{C}$. Thus, from (3.4), we can conclude that $\left.\mathcal{F}_{S} \mu\right|_{\mathcal{C}}=0$.

Remark 3.2. (a) Further, we observed that Theorem 3.1 holds for a nonharmonic real cone. Let $\mathcal{C}$ be a non-harmonic real cone. Write $\tilde{\omega}=\sigma_{o} \omega$, where $\sigma_{o}$ is the symplectic matrix, which in fact, belongs to $U(n) \subset S O(2 n)$. Suppose $\mu \in X\left(S^{2 n-1}\right)$ satisfies $\left.\mathcal{F}_{S} \mu\right|_{\mathcal{C}}=0$. Then $\Pi_{l} f\left(\sigma_{o} \omega\right)=0$ for all $l \in \mathbb{Z}_{+}$. Since $\sigma_{o}^{-1} \cdot \Pi_{l} f$ would also be a spherical harmonic, we infer that $\left(S^{2 n-1}, \mathcal{C}\right)$ is a HUP for SFT.

(b) Suppose $\Gamma$ be a smooth sub-manifold in $\mathbb{R}^{2 n}$ and $\Lambda$ be a subset of $\mathbb{R}^{2 n}$. Let $T: \mathbb{R}^{2 n} \rightarrow \mathbb{R}^{2 n}$ be defined by $T(x, y)=\left(\frac{y}{2},-\frac{x}{2}\right)$ for $x, y \in \mathbb{R}^{n}$. It is easy to see that $\mathcal{F}_{S} \mu(x, y)=\hat{\mu}\left(\frac{y}{2},-\frac{x}{2}\right)$, where $\hat{\mu}$ is the Euclidean Fourier transform (EFT) of $\mu$. Thus, $(\Gamma, \Lambda)$ is a HUP for SFT if and only if $(\Gamma, T \Lambda)$ is a HUP for the EFT.

For instance, by the Euclidean result ([45], Proposition 1.2), $\left(S^{2 n-1}, S_{r}^{2 n-1}\right)$ is a HUP for the SFT as long as $\frac{r}{2} \notin J_{(n+k-1)}^{-1}(0)$ for any $k \in \mathbb{Z}_{+}$.

(c) Consider the map $\tilde{T}:=\left(T^{-1}\right)^{*}$ on $\mathbb{R}^{2 n}$. It is known for the EFT that $(\Gamma, \Lambda)$ is a HUP if and only if $\left(\tilde{T}^{-1} \Gamma, \tilde{T}^{*} \Lambda\right)$ is a HUP. Hence, from Remark $(b)$, we have $(\Gamma, \Lambda)$ is a HUP for SFT if and only if $\left(T^{*} \Gamma, \Lambda\right)$ is a HUP for EFT. Thus, in view of the Euclidean result ([40], Theorem 3.1), Remark (a) holds true.

\section{UNIQUENESS PAIRS FOR THE MODIFIED FOURIER TRANSFORM ON $\mathbb{H}^{n}$}

In this section, we prove that a finite measure supported on the cylinder $S_{r}^{2 n-1} \times \mathbb{R}$ can be determined by a non-harmonic cone as well as by the boundary of a bounded domain in $\mathbb{C}^{n}$.

We know from [42] that the modified Fourier transform of $f \in L^{1}\left(\mathbb{H}^{n}\right)$ is defined by

$$
\mathcal{F}_{M} f(z, \lambda)=\pi_{\lambda}(-z) W_{\lambda}\left(f^{\lambda}\right) \pi_{\lambda}(z),
$$

where $W_{\lambda}\left(f^{\lambda}\right)$ is the Weyl transform of $f^{\lambda}$ and $(z, \lambda) \in \mathbb{C}^{n} \times \mathbb{R}^{*}$. This, in turn, can be expressed as

$$
\begin{aligned}
\mathcal{F}_{M} f(z, \lambda) & =\int_{\mathbb{C}^{n}} \pi_{\lambda}(-z) \pi_{\lambda}(w) f^{\lambda}(w) \pi_{\lambda}(z) d w \\
& =\int_{\mathbb{C}^{n}} e^{-i \lambda \operatorname{Im}(z \cdot \bar{w})} f^{\lambda}(w) \pi_{\lambda}(w) d w .
\end{aligned}
$$


Consider the measure $\mu \in X\left(S_{r}^{2 n-1} \times \mathbb{R}\right)$. Then there exists $f \in L^{1}\left(S_{r}^{2 n-1} \times \mathbb{R}\right)$ such that $d \mu(\zeta, t)=f(\zeta, t) d \sigma_{r}(\zeta) d t$. For $(z, \lambda) \in \mathbb{C}^{n} \times \mathbb{R}^{*}$, define the modified Fourier transform of $\mu$ by

$$
\mathcal{F}_{M} \mu(z, \lambda)=\int_{S_{r}^{2 n-1}} e^{-i \lambda \operatorname{Im}(z \cdot \bar{\zeta})} f^{\lambda}(\zeta) \pi_{\lambda}(\zeta) d \sigma_{r}(\zeta)
$$

For $\lambda \in \mathbb{R}^{*}$, consider the subspace $U_{\lambda}=\operatorname{span}\left\{\phi_{\alpha_{\lambda}}^{\lambda}\right\}$ of $L^{2}\left(\mathbb{R}^{n}\right)$, where $\phi_{\alpha_{\lambda}}^{\lambda}$ is the scaled Hermite function for some $\alpha_{\lambda} \in \mathbb{Z}_{+}^{n}$. Denote $\tilde{\Lambda}=\mathcal{C} \times \mathbb{R}^{*}$, where $\mathcal{C}$ could be a real (or complex) cone. We have proved the following result.

Proposition 4.1. Let $\mu \in X\left(S_{r}^{2 n-1} \times \mathbb{R}\right)$ and range of $\mathcal{F}_{M} \mu(\xi, \lambda)$ is a subspace of $U_{\lambda}^{\perp}$ for all $(\xi, \lambda) \in \tilde{\Lambda}$. If $\mathcal{C}$ is non-harmonic, then $\mu=0$.

Proof. Since, for each $\lambda \in \mathbb{R}^{*}$, range of $\mathcal{F}_{M} \mu(\xi, \lambda)$ is a subspace of $U_{\lambda}^{\perp}$, it follows that

$$
\left\langle\mathcal{F}_{M} \mu(z, \lambda) \varphi, \phi_{\alpha_{\lambda}}^{\lambda}\right\rangle=0
$$

for all $\varphi \in L^{2}\left(\mathbb{R}^{n}\right)$. We know from 42 that $\left\langle\pi_{\lambda}(w) \phi_{o}^{\lambda}, \phi_{\alpha}^{\lambda}\right\rangle=c_{\alpha}|\lambda|^{|\alpha| / 2} w^{\alpha} e^{\frac{-|\lambda||w|^{2}}{4}}$ for all $w \in \mathbb{C}^{n}$. If we choose $\varphi=\phi_{o}^{\lambda}$, then from (4.1) we have

$$
\int_{S_{r}^{2 n-1}} e^{-i \lambda \operatorname{Im}(z \cdot \bar{\zeta})} f^{\lambda}(\zeta) c_{\alpha_{\lambda}} \zeta^{\alpha_{\lambda}} e^{\frac{-|\lambda||\zeta|^{2}}{4}} d \sigma_{r}(\zeta)=0
$$

for all $z \in \mathcal{C}$. This, in fact, reduces to the case of SFT on $\mathbb{C}^{n}$. That is, for each $\lambda \in \mathbb{R}^{*}$, we get $\mathcal{F}_{S}\left(g_{r}^{\lambda}\right)(2 r \lambda z)=0$ for all $z \in \mathcal{C}$, where $g_{r}^{\lambda}(\nu)=f^{\lambda}(r \nu) \nu^{\alpha_{\lambda}}$ for $\nu \in S^{2 n-1}$. Since $(2 r \lambda) \mathcal{C} \subseteq \mathcal{C}$, in view of Theorem 3.1 and Remark 3.2 (a), we infer that $f^{\lambda}=0$ if and only if $\mathcal{C}$ is non-harmonic. Thus, $f=0$.

Remark 4.2. Let $\mu \in X(\Gamma \times \mathbb{R})$ and range of $\mathcal{F}_{M} \mu(z, \lambda)$ is a subspace of $U_{\lambda}^{\perp}$, whenever $(z, \lambda) \in \Lambda \times \mathbb{R}^{*}$. If $(\Gamma, s T \Lambda)$ is a HUP for EFT for almost all $s \in \mathbb{R}$, then Remark $3.2(b)$ and Equation (4.2) allow to conclude that $\mu=0$.

For instance, consider $\Gamma=S^{2 n-1}$ and $\Lambda=S_{r}^{2 n-1}$. Since the set $\left\{J_{n+k-1}^{-1}(0)\right.$ : $\left.k \in \mathbb{Z}_{+}\right\}$has measure zero, in view of the Euclidean result (45], Proposition 1.2), we conclude $\mu=0$.

Theorem 4.3. Let $\partial \Omega$ be the boundary of the bounded domain $\Omega$ in $\mathbb{C}^{n}$. If $\mu \in X\left(S_{r}^{2 n-1} \times \mathbb{R}\right)$ satisfies $\mathcal{F}_{M} \mu(\xi, \lambda)=0$ for all $(\xi, \lambda) \in \partial \Omega \times \mathbb{R}^{*}$, then $\mu=0$.

Proof. Since $\mathcal{F}_{M} \mu$ can be extended holomorphically to a function $F(., \lambda)$ on $\mathbb{C}^{2 n}$, taking values in $L^{2}\left(\mathbb{R}^{n}\right)$, it follows that $\left.F(., \lambda)\right|_{\mathbb{R}^{2 n}}=\mathcal{F}_{M} \mu$ is a real analytic function. Consider

$$
\mathcal{F}_{M} \mu(z, \lambda)=\int_{S_{r}^{2 n-1}} e^{-i \lambda \operatorname{Im}(z \cdot \bar{\zeta})} f^{\lambda}(\zeta) \pi_{\lambda}(\zeta) d \sigma_{r}(\zeta) .
$$

Then

$$
\frac{\partial}{\partial z_{j}} \mathcal{F}_{M} \mu(z, \lambda)=\frac{\lambda}{2} \int_{S_{r}^{2 n-1}} \bar{\zeta}_{j} e^{-i \lambda \operatorname{Im}(z \cdot \bar{\zeta})} f^{\lambda}(\zeta) \pi_{\lambda}(\zeta) d \sigma_{r}(\zeta)
$$


and

$$
\frac{\partial^{2}}{\partial \bar{z}_{j} \partial z_{j}} \mathcal{F}_{M} \mu(z, \lambda)=-\frac{\lambda^{2}}{4} \int_{S_{r}^{2 n-1}} \bar{\zeta}_{j} \zeta_{j} e^{-i \lambda \operatorname{Im}(z \cdot \bar{\zeta})} f^{\lambda}(\zeta) \pi_{\lambda}(\zeta) d \sigma_{r}(\zeta) .
$$

This, in turn, implies

$$
\triangle_{z} \mathcal{F}_{M} \mu(z, \lambda)+(r \lambda)^{2} \mathcal{F}_{M} \mu(z, \lambda)=0 .
$$

Now, if $\varphi, \psi \in L^{2}\left(\mathbb{R}^{n}\right)$, then we have

$$
\triangle_{z}\left\langle\mathcal{F}_{M} \mu(z, \lambda) \varphi, \psi\right\rangle+(r \lambda)^{2}\left\langle\mathcal{F}_{M} \mu(z, \lambda) \varphi, \psi\right\rangle=0 .
$$

Let $g(z, \lambda)=\left\langle\mathcal{F}_{M} \mu(z, \lambda) \varphi, \psi\right\rangle$. Then $g$ will be a real analytic function satisfying

$$
\triangle_{\xi} g(z, \lambda)+(r \lambda)^{2} g(z, \lambda)=0 .
$$

Hence $g(., \lambda) ; \lambda \in \mathbb{R}^{*}$ are eigenfunctions of the Dirichlet boundary value problem in $\mathbb{C}^{n}$. By the discreetness of eigenvalues of the Dirichlet problem in the bounded domain, it follows that $g(., \lambda)=0$ for all most all $\lambda \in \mathbb{R}^{*}$. Since $g(., \lambda)$ is continuous in $\lambda$, we infer that $g(z, \lambda)=0$ for all $(z, \lambda) \in \mathbb{C}^{n} \times \mathbb{R}^{*}$. Thus, $\mu=0$.

Remark 4.4. Let $u$ be a solution of the Helmholtz equation $\triangle u+c^{2} u=0$ on $\mathbb{R}^{2 n}$ and $\Sigma \subset \mathbb{R}^{2 n}$ be such that $u=0$ on $\Sigma$ implies $u=0$ a.e. Then the statment of Theorem 4.3 will remain true if we replace $\partial \Omega$ by $\Sigma$. Regular Jordan curves and two intersecting curves separated by angle which is an irrational multiple of $\pi$, are examples of such $\Sigma$. For details, see [13].

\section{UNIQUENESS PAIRS FOR THE SPECTRAL PROJECTIONS}

In this section, we derive that sphere is determining set for the spectral projections of finite measures on $\mathbb{C}^{n}$, which are supported on $S^{2 n-1}$. Further, we deduce that non-harmonic complex cone as well as $N A$-set can determine the spectral projections of the above class of measures.

For $\mu \in X\left(S_{r}^{2 n-1}\right)$, we define the spectral projection of $\mu$ by

$$
\varphi_{k}^{n-1} \times \mu(z)=\int_{S_{r}^{2 n-1}} \varphi_{k}^{n-1}(z-\omega) e^{\frac{i}{2} \operatorname{Im}(z \cdot \bar{\omega})} d \mu(\omega) .
$$

Then the following result holds true.

Theorem 5.1. Let $\mu \in X\left(S_{r_{1}}^{2 n-1}\right)$ be such that $\varphi_{k}^{n-1} \times \mu(z)=0$ for all $z \in S_{r_{2}}^{2 n-1}$ and $k \in \mathbb{Z}_{+}$. Then $\mu=0$.

In order to prove Theorem 5.1, we need the following results about irreducibility of the Laguerre polynomials.

Theorem 5.2. [14] Let $v$ be a rational number, which is not a negative integer. Then for all but finitely many $k \in \mathbb{Z}_{+}$, the Laguerre polynomial $L_{k}^{v}$ is irreducible over the field of rationals. 
Notice that the disjointness of the zero set of Laguerre functions can be understood by the following description of the zero set of Laguerre polynomials. This follows from Theorem [5.2, and has worked out in 38.

Proposition 5.3. 38 Let $k \in \mathbb{Z}_{+}$. Then for all but finitely many $k$, the Laguerre polynomials $L_{k}^{n-1}$ have distinct zeroes over the reals.

Proof of Theorem 5.1. Since $\mu \in X\left(S_{r_{1}}^{2 n-1}\right)$, there exists $f \in L^{1}\left(S_{r_{1}}^{2 n-1}\right)$ such that $d \mu=f d \sigma_{r_{1}}$. Thus,

$$
\varphi_{k}^{n-1} \times \mu(z)=\int_{S_{r_{1}}^{2 n-1}} \varphi_{k}^{n-1}(z-\omega) e^{\frac{i}{2} \operatorname{Im}(z \cdot \bar{\omega})} f(\omega) d \sigma_{r_{1}}(\omega)=0,
$$

for all $k \in \mathbb{Z}_{+}$and $z \in S_{r_{2}}^{2 n-1}$. As $f \in L^{1}\left(S_{r_{1}}^{2 n-1}\right), f$ will satisfy

$$
f=\lim _{m \rightarrow \infty} \sum_{l=0}^{m} A_{l}^{m}(\delta) \Pi_{l} f
$$

where $A_{l}^{m}(\delta)=\left(\begin{array}{c}m-l+\delta \\ \delta\end{array}\right)\left(\begin{array}{c}m+\delta \\ \delta\end{array}\right)^{-1}$ and $\delta>n-1$. Further, from Lemma 2.2, it follows that

$$
f=\lim _{m \rightarrow \infty} \sum_{l=0}^{m} \sum_{p+q=l} A_{p+q}^{m}(\delta) \Pi_{p, q} f .
$$

Now, from condition (5.1), it follows that

$$
\begin{aligned}
\left|\sum_{l=0}^{m} \sum_{p+q=l} A_{p+q}^{m}(\delta) \varphi_{k}^{n-1} \times \Pi_{p, q} f(z)\right| & =\left|\sum_{l=0}^{m} \sum_{p+q=l} A_{p+q}^{m}(\delta) \varphi_{k}^{n-1} \times \Pi_{p, q} f(z)-\varphi_{k}^{n-1} \times f(z)\right| \\
\leq & M_{k} \int_{S_{r_{1}}^{2 n-1}}\left|\sum_{l=0}^{m} \sum_{p+q=l} A_{p+q}^{m}(\delta) \Pi_{p, q} f(\omega)-f(\omega)\right| d \sigma_{r_{1}}(\omega),
\end{aligned}
$$

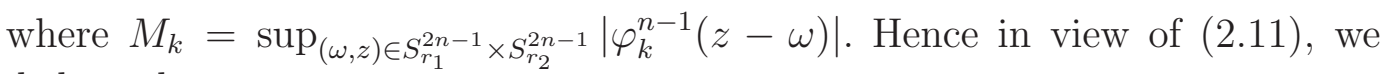
deduce that

$$
\lim _{m \rightarrow \infty} \sum_{l=0}^{m} \sum_{p+q=l} A_{p+q}^{m}(\delta) \varphi_{k}^{n-1} \times \Pi_{p, q} f=0
$$

converges uniformly in $S_{r_{2}}^{2 n-1}$, whenever $k \in \mathbb{Z}_{+}$. When $k \geq q$, Lemma 2.1 gives

$$
\int_{S^{2 n-1}} \varphi_{k}^{n-1}\left(z-r_{1} \eta\right) e^{\frac{i}{2} r_{1} \operatorname{Im}(z \cdot \bar{\eta})} Y_{p, q}(\eta) d \sigma(\eta)=B_{n}^{k, \gamma} r_{1}^{p+q} \varphi_{k-q}^{\gamma-1}\left(r_{1}\right) \varphi_{k-q}^{\gamma-1}(z) P_{p, q}(z),
$$


where $B_{n}^{k, \gamma}=(2 \pi)^{-n} \frac{\Gamma(k-q+1)}{\Gamma(k+n+p)}$ and $\gamma=n+p+q$. Let $z=r_{2} \xi$, where $\xi \in S^{2 n-1}$. Then from (5.2) and (5.3), we infer that

$$
\lim _{m \rightarrow \infty} \sum_{l=0}^{m} \sum_{p+q=l} A_{p+q}^{m}(\delta) B_{n}^{k, \gamma}\left(r_{1} r_{2}\right)^{p+q} \varphi_{k-q}^{\gamma-1}\left(r_{1}\right) \varphi_{k-q}^{\gamma-1}\left(r_{2}\right) \Pi_{p, q} f(\xi)=0 .
$$

Since (5.2) converges uniformly on $S_{r_{2}}^{2 n-1}$, it follows that (5.4) converges in $L^{2}\left(S^{2 n-1}\right)$. Recall that the bi-gradted spherical harmonic projections $\Pi_{p, q} f$ are orthogonal among themselves, and $A_{p+q}^{m}(\delta) B_{n}^{k, \gamma} \neq 0$ holds true for every choice of $p, q \in \mathbb{Z}_{+}$. From (5.4) we obtain that

$$
\varphi_{k-q}^{\gamma-1}\left(r_{1}\right) \varphi_{k-q}^{\gamma-1}\left(r_{2}\right)\left\|\Pi_{p, q} f\right\|_{2}=0,
$$

whenever $k \geq q$. Hence by invoking Proposition 5.3 for each pair of $p, q \in \mathbb{Z}_{+}$, there exists $k_{o} \geq q$ such that $r_{i} \notin\left(\varphi_{k_{o}-q}^{n+p+q-1}\right)^{-1}(0)$; when $i=1,2$. Hence, from (5.5) we conclude that $\Pi_{p, q} f=0$ for all $p, q \in \mathbb{Z}_{+}$. Thus, $f=0$.

Remark 5.4. A set, which is a determining set for any real analytic function, is called $N A$ - set. For instance, the spiral is an $N A$ - set in the plane (see [30]). Since the spectral projection $\varphi_{k}^{n-1} \times \mu$ can be extended holomorphically to $\mathbb{C}^{2 n}$, the function $\varphi_{k}^{n-1} \times \mu$ must be real analytic on $\mathbb{C}^{n}$.

Let $\Lambda$ be an NA-set for real analytic functions on $\mathbb{C}^{n}$. If $\mu \in X\left(S_{r}^{2 n-1}\right)$ satisfies $\varphi_{k}^{n-1} \times\left.\mu\right|_{\Lambda}=0$ for all $k \in \mathbb{Z}_{+}$, then $\varphi_{k}^{n-1} \times \mu(z)=0$ for all $z \in \mathbb{C}^{n}$. Thus, in view of Theorem 5.1, we have $f=0$.

Next, we shall prove that spectral projections of a measure $\mu \in X\left(S_{r}^{2 n-1}\right)$ can be determined by a non-harmonic complex cone.

Theorem 5.5. Let $\mathcal{C}$ be a non-harmonic complex cone in $\mathbb{C}^{n}$. If $\mu \in X\left(S_{r}^{2 n-1}\right)$ satisfies $\varphi_{k}^{n-1} \times\left.\mu\right|_{\mathcal{C}}=0$ for all $k \in \mathbb{Z}_{+}$, then $\mu=0$.

Proof. From (5.4), it follows that

$$
\lim _{m \rightarrow \infty} \sum_{l=0}^{m} \sum_{p+q=l} A_{p+q}^{m}(\delta) B_{n}^{k, \gamma}(r s)^{p+q} \varphi_{k-q}^{\gamma-1}(r) \varphi_{k-q}^{\gamma-1}(s) \Pi_{p, q} f(\xi)=0,
$$

whenever $s \xi \in \mathcal{C}$ and $k \in \mathbb{Z}_{+}$. Since $\mathcal{C}$ is closed under complex scaling, replacing $\xi$ by $e^{i \theta} \xi$, we obtain

$$
\lim _{m \rightarrow \infty} \sum_{l=0}^{m} \sum_{p+q=l} A_{p+q}^{m}(\delta) B_{n}^{k, \gamma}(r s)^{p+q} \varphi_{k-q}^{\gamma-1}(r) \varphi_{k-q}^{\gamma-1}(s) \Pi_{p, q} f(\xi) e^{i(p-q) \theta}=0 .
$$

Now, by induction on $k$, we show that each of the projection $\Pi_{p, q} f$ restricted to $\mathcal{C}$ must be zero. For $k=0$, the choice for $q$ is only 0 . Since $\left\{e^{i p \theta}: p \in \mathbb{Z}_{+}\right\}$ is an orthogonal set, we infer that $\Pi_{p, 0}(f)(\xi)=0$. Similarly, for $k=1$, the choices for $q$ are 0 and 1 only. The case $q=0$ is already settled. Now for $q=1,\left\{e^{i(p-1) \theta}: p \in \mathbb{Z}_{+}\right\}$is an orthogonal set. Hence $\Pi_{p, 1}(f)(\xi)=0$. This, in turn, implies that each of $\Pi_{p, q}(f)$ vanishes on $\mathcal{C}$. Thus $f=0$. 


\section{Benedicks-Amrein-Berthier theorem}

In this section, we prove Benedicks-Amrein-Berthier theorem for the Heisenberg group.

For $\lambda=1$, we denote $W_{1}(g)$ by $W(g)$. For $g \in L^{2}\left(\mathbb{C}^{n}\right)$, suppose $W(g)$ is of finite rank. Then there exists an orthonormal basis $\left\{e_{1}, e_{2}, \ldots\right\}$ of $L^{2}\left(\mathbb{R}^{n}\right)$ such that $\mathcal{R}(W(g))=\mathcal{B}_{N}$, where $\mathcal{B}_{N}=\operatorname{span}\left\{e_{1}, \ldots, e_{N}\right\}$ and $\mathcal{R}$ stands for the range. Define an orthogonal projection $P_{N}$ of $L^{2}\left(\mathbb{R}^{n}\right)$ onto $\mathcal{B}_{N}$. Let $A$ be a measurable subset of $\mathbb{C}^{n}$. Define a pair of orthogonal projections $E_{A}$ and $F_{N}$ of $L^{2}\left(\mathbb{C}^{n}\right)$ by

$$
E_{A} g=\chi_{A} g \quad \text { and } \quad W\left(F_{N} g\right)=P_{N} W(g),
$$

where $\chi_{A}$ denotes the characteristic function of $A$. Then $\mathcal{R}\left(E_{A}\right)=\{g \in$ $\left.L^{2}\left(\mathbb{C}^{n}\right): g=\chi_{A} g\right\}$ and $\mathcal{R}\left(F_{N}\right)=\left\{g \in L^{2}\left(\mathbb{C}^{n}\right): \mathcal{R}(W(g)) \subseteq \mathcal{B}_{N}\right\}$.

First, we prove that $E_{A} F_{N}$ is a Hilbert-Schmidt operator that satisfies $\left\|E_{A} F_{N}\right\|_{H S}^{2}=(2 \pi)^{-n} m(A) N$. Throughout this section, $A$ will be considered as a set of finite Lebesgue measure.

Lemma 6.1. The operator $E_{A} F_{N}$ is an integral operator with kernel $K(z, w)=$ $(2 \pi)^{-n} \chi_{A}(z) \operatorname{tr}\left(P_{N} \pi(w) \pi(-z)\right)$, where $z, w \in \mathbb{C}^{n}$.

Proof. For $g \in L^{2}\left(\mathbb{C}^{n}\right)$, we have $W\left(F_{N} g\right)=P_{N} W(g)$. By inversion formula for the Weyl transform, we have

$$
\begin{aligned}
\left(F_{N} g\right)(z) & =(2 \pi)^{-n} \operatorname{tr}\left(\pi(z)^{*} W\left(F_{N} g\right)\right)=(2 \pi)^{-n} \operatorname{tr}\left(\pi(-z) P_{N} W(g)\right) \\
& =(2 \pi)^{-n} \operatorname{tr}\left(P_{N} W(g) \pi(-z)\right) \\
& =(2 \pi)^{-n} \int_{\mathbb{C}^{n}} g(w) \operatorname{tr}\left(P_{N} \pi(w) \pi(-z)\right) d w .
\end{aligned}
$$

Hence, we can write

$$
\begin{aligned}
\left(E_{A} F_{N} g\right)(z) & =\chi_{A}(z)\left(F_{N} g\right)(z)=(2 \pi)^{-n} \chi_{A}(z) \int_{\mathbb{C}^{n}} g(w) \operatorname{tr}\left(P_{N} \pi(w) \pi(-z)\right) d w \\
& =\int_{\mathbb{C}^{n}} g(w) K(z, w) d w
\end{aligned}
$$

where $K(z, w)=(2 \pi)^{-n} \chi_{A}(z) \operatorname{tr}\left(P_{N} \pi(w) \pi(-z)\right)$. By this, we infer that $E_{A} F_{N}$ is an integral operator with kernel $K$.

Lemma 6.2. $E_{A} F_{N}$ is Hilbert-Schmidt and $\left\|E_{A} F_{N}\right\|_{H S}^{2}=(2 \pi)^{-n} m(A) N$. 
Proof. From Lemma 6.1, we know that $E_{A} F_{N}$ is an integral operator with kernel $K(z, w)$. Therefore,

$$
\begin{aligned}
\left\|E_{A} F_{N}\right\|_{H S}^{2} & =\int_{\mathbb{C}^{n}} \int_{\mathbb{C}^{n}}|K(z, w)|^{2} d w d z \\
& =(2 \pi)^{-2 n} \int_{\mathbb{C}^{n}}\left|\chi_{A}(z)\right|^{2}\left(\int_{\mathbb{C}^{n}}\left|\operatorname{tr}\left(P_{N} \pi(w) \pi(-z)\right)\right|^{2} d w\right) d z \\
& =(2 \pi)^{-2 n} \int_{\mathbb{C}^{n}} \chi_{A}(z)\left(\int_{\mathbb{C}^{n}}\left|\sum_{j=1}^{N}\left\langle\pi(w) \pi(-z) e_{j}, e_{j}\right\rangle\right|^{2} d w\right) d z
\end{aligned}
$$

Since $\pi(w) \pi(z)=e^{\frac{i}{2} \operatorname{Im}(w \cdot \bar{z})} \pi(w+z)$, we get

$$
\begin{aligned}
\left\|E_{A} F_{N}\right\|_{H S}^{2} & =(2 \pi)^{-2 n} \int_{\mathbb{C}^{n}} \chi_{A}(z) \int_{\mathbb{C}^{n}}\left|e^{-\frac{i}{2} \operatorname{Im}(w \cdot \bar{z})} \sum_{j=1}^{N}\left\langle\pi(w-z) e_{j}, e_{j}\right\rangle\right|^{2} d w d z \\
& =(2 \pi)^{-2 n} \int_{\mathbb{C}^{n}} \chi_{A}(z) \int_{\mathbb{C}^{n}}\left|\sum_{j=1}^{N}\left\langle\pi(w) e_{j}, e_{j}\right\rangle\right|^{2} d w d z .
\end{aligned}
$$

Hence, from the orthogonality relation (2.2) of the Fourier-Winger transform, the lemma will be followed.

We need the following result from [2] that describes an interesting property of measurable sets having finite measure. Denote $w A=\left\{z \in \mathbb{C}^{n}: z-w \in A\right\}$.

Lemma 6.3. 2] Let $B$ be a measurable set in $\mathbb{C}^{n}$ with $0<m(B)<\infty$. If $B_{0}$ is a measurable subset of $B$ with $m\left(B_{0}\right)>0$, then for $\epsilon>0$, there exists $w \in \mathbb{C}^{n}$ such that

$$
m(B)<m\left(B \cup w B_{0}\right)<m(B)+\epsilon .
$$

Now, for orthogonal projections $E$ and $F$ on a Hilbert space $\mathcal{H}$, let $E \cap F$ denote the orthogonal projection of $\mathcal{H}$ onto $\mathcal{R}(E) \cap \mathcal{R}(F)$. Then, we have the relation

$$
\|E \cap F\|_{H S}^{2}=\operatorname{dim} \mathcal{R}(E \cap F) \leq\|E F\|_{H S}^{2} .
$$

We abbreviate $A^{c}=\mathbb{C}^{n} \backslash A$ and $F_{N}^{\perp}=I-F_{N}$. Let $S$ be a closed subspace of $L^{2}\left(\mathbb{R}^{n}\right)$. Define $F_{S}$ by $W\left(F_{S} g\right)=P_{S} W(g)$, where $P_{S}$ is the orthogonal projection of $L^{2}\left(\mathbb{R}^{n}\right)$ onto $S$ and $g \in L^{2}\left(\mathbb{C}^{n}\right)$. In particular, if $S=\mathcal{B}_{N}$, then $F_{S}=F_{N}$.

Proposition 6.4. Let $A \subset \mathbb{C}^{n}$ with finite measure, and $S$ be a closed subspace of $L^{2}\left(\mathbb{R}^{n}\right)$. Then, either $E_{A} \cap F_{S}=0$ or for every $\epsilon^{\prime}>0$, there exists $\tilde{A} \supset A$ with $m(\tilde{A} \backslash A)<\epsilon^{\prime}$ such that $\mathcal{R}\left(E_{\tilde{A}} \cap F_{S}\right)$ is of infinite dimensional.

Proof. If $E_{A} \cap F_{S} \neq 0$, then there exists a non-zero function $g_{0} \in \mathcal{R}\left(E_{A} \cap F_{S}\right)$. Let $A_{0}=\left\{x \in A: g_{0}(x) \neq 0\right\}$ and $\tilde{A}_{1}=A$. By Lemma 6.3, for $\epsilon=\frac{\epsilon^{\prime}}{2^{l}}, B_{0}=A_{0}$ 
and $B=\tilde{A}_{l}$, there exists $w_{l} \in \mathbb{C}^{n}$ such that

$$
m\left(\tilde{A}_{l}\right)<m\left(\tilde{A}_{l} \cup w_{l} A_{0}\right)<m\left(\tilde{A}_{l}\right)+\frac{\epsilon^{\prime}}{2^{l}} .
$$

Put $\tilde{A}_{l+1}=\tilde{A}_{l} \cup w_{l} A_{0}$ and $\tilde{A}=\bigcup_{l=1}^{\infty} \tilde{A}_{l}$. Then $\tilde{A}_{l}$ is a non-decreasing sequence, and hence from (6.3) it follows that $m(\tilde{A} \backslash A)<\epsilon^{\prime}$. For $l \in \mathbb{N}$, consider $g_{l}(z)=e^{\frac{i}{2} \operatorname{Im}\left(z \cdot \bar{w}_{l}\right)} g_{0}\left(z-w_{l}\right)$. We show that $g_{l} \in \mathcal{R}\left(E_{\tilde{A}} \cap F_{S}\right)$ for each $l \in \mathbb{N}$, and they are linearly independent. Let $\mathcal{B}_{S}$ be an orthonormal basis of $S$. Then we can extend $\mathcal{B}_{S}$ to an orthonormal basis $\mathcal{B}$ of $L^{2}\left(\mathbb{R}^{n}\right)$. For $\varphi \in L^{2}\left(\mathbb{R}^{n}\right)$ and $\psi \in \mathcal{B} \backslash \mathcal{B}_{S}$, we have

$$
\begin{aligned}
\left\langle W\left(g_{l}\right) \varphi, \psi\right\rangle & =\int_{\mathbb{C}^{n}} g_{l}(z)\langle\pi(z) \varphi, \psi\rangle d z \\
& =\int_{\mathbb{C}^{n}} e^{\frac{i}{2} \operatorname{Im}\left(z \cdot \bar{w}_{l}\right)} g_{0}\left(z-w_{l}\right)\langle\pi(z) \varphi, \psi\rangle d z \\
& =\int_{\mathbb{C}^{n}} e^{\frac{i}{2} \operatorname{Im}\left(z \cdot \bar{w}_{l}\right)} g_{0}(z)\left\langle\pi\left(z+w_{l}\right) \varphi, \psi\right\rangle d z .
\end{aligned}
$$

Since $\pi(z) \pi(w)=e^{\frac{i}{2} \operatorname{Im}(z \cdot \bar{w})} \pi(z+w)$, we get

$$
\begin{aligned}
\left\langle W\left(g_{l}\right) \varphi, \psi\right\rangle & =\int_{\mathbb{C}^{n}} g_{0}(z)\left\langle\pi(z) \pi\left(w_{l}\right) \varphi, \psi\right\rangle d z \\
& =\int_{\mathbb{C}^{n}} g_{0}(z)\langle\pi(z) \tilde{\varphi}, \psi\rangle d z \\
& =\left\langle W\left(g_{0}\right) \tilde{\varphi}, \psi\right\rangle=0 .
\end{aligned}
$$

Hence $\mathcal{R}\left(W\left(g_{l}\right)\right) \subseteq \mathcal{B}_{S}$. Let $A_{l}=A_{l-1} \cup w_{l} A_{0}$. Then $\tilde{A}_{l+1}=\tilde{A}_{l} \cup A_{l}$. Thus, $m\left(A_{l} \backslash A_{l-1}\right) \geq m\left(\tilde{A}_{l+1} \backslash \tilde{A}_{l}\right)>0$. Let $s \in \mathbb{N}$. Since, $A_{s}=A_{0} \cup w_{1} A_{0} \cup \cdots \cup w_{s} A_{0}$ and $g_{l}(z)=0$ on $\left(w_{l} A_{0}\right)^{c}$, we have $E_{A_{s}} g_{l}=g_{l}$ for $l=0,1, \ldots, s$. Furthermore, $E_{A_{s} \backslash A_{s-1}} g_{l}=0$ for $l=0, \ldots, s-1$ and $E_{A_{s} \backslash A_{s-1}} g_{s} \neq 0$. Therefore, it shows that $g_{s}$ is not a linear combination of $g_{0}, \ldots, g_{s-1}$. Since $s$ is arbitrary, $\left\{g_{l}\right.$ : $l \in \mathbb{N}\}$ is a linearly independent set in $\mathcal{R}\left(E_{\tilde{A}} \cap F_{S}\right)$. This proves the required proposition.

Proposition 6.5. Let $A$ be a measurable subset of $\mathbb{C}^{n}$ having finite Lebesgue measure. Then the projection $E_{A} \cap F_{N}=0$.

Proof. In view of (6.2) and Lemma 6.2, we obtain

$$
\operatorname{dim} \mathcal{R}\left(E_{\tilde{A}} \cap F_{N}\right) \leq(2 \pi)^{-n} m(\tilde{A}) N<\infty .
$$

Therefore, as a corollary of Proposition 6.4, we get $E_{A} \cap F_{N}=0$.

Remark 6.6. If $0<m(A)<\infty$, then $\operatorname{dim} \mathcal{R}\left(E_{A}\right)=\infty$. Now, in view of Proposition 6.5 and the fact that $E_{A}=\left(E_{A} \cap F_{N}\right)+\left(E_{A} \cap F_{N}^{\perp}\right)=\left(E_{A} \cap F_{N}^{\perp}\right)$, it follows that $\operatorname{dim} \mathcal{R}\left(E_{A} \cap F_{N}^{\perp}\right)=\infty$. Since $m\left(A^{c}\right)=\infty$, there exists a measurable 
set $B \subseteq A^{c}$ satisfying $0<m(B)<\infty$. Hence $\mathcal{R}\left(E_{A^{c}} \cap F_{N}^{\perp}\right) \supseteq \mathcal{R}\left(E_{B} \cap F_{N}^{\perp}\right)$. This implies $\operatorname{dim} \mathcal{R}\left(E_{A^{c}} \cap F_{N}^{\perp}\right)=\infty$. Similarly, $\operatorname{dim} \mathcal{R}\left(E_{A^{c}} \cap F_{N}\right)=\infty$.

The following theorem is our main result of this section, which is analogous to Benedicks-Amrein-Berthier theorem for the Heisenberg group.

Theorem 6.7. Let $A \subset \mathbb{C}^{n}$ be a set of finite Lebesgue measure. Suppose $f \in L^{1}\left(\mathbb{H}^{n}\right)$ and $\left\{(z, t) \in \mathbb{H}^{n}: f(z, t) \neq 0\right\} \subseteq A \times \mathbb{R}$. If $\hat{f}(\lambda)$ is a finite rank operator for each $\lambda \in \mathbb{R}^{*}$, then $f=0$.

In order to prove Theorem 6.7, it is sufficient to prove the following result for the Weyl transform $W_{\lambda}$.

Proposition 6.8. Let $g \in L^{1}\left(\mathbb{C}^{n}\right)$ and $\left\{z \in \mathbb{C}^{n}: g(z) \neq 0\right\} \subseteq A$, where $m(A)$ is finite. Let $\lambda \in \mathbb{R}^{*}$ and $W_{\lambda}(g)$ has finite rank. Then $g=0$.

Since $W_{\lambda}(g)$ is a finite rank operator, by the Plancheral theorem for the Weyl transform, $g \in L^{2}\left(\mathbb{C}^{n}\right)$. Thus, it is enough to prove Proposition 6.8 for $g \in L^{2}\left(\mathbb{C}^{n}\right)$ and $\lambda=1$. Note that Proposition 6.8 follows from Proposition 6.5.

Remark 6.9. As similar to the Euclidean set up, for strong annihilating pair (SAP) [20], Proposition 6.8 leads to the following concept of strong annihilating pair for the Weyl transform, which appears in terms of support of the function and rank of its Weyl transform.

Let $A$ be a measurable subset of $\mathbb{C}^{n}$ and $P_{S}$ be the orthogonal projection of $L^{2}\left(\mathbb{R}^{n}\right)$ onto a closed subspace $S$ of $L^{2}\left(\mathbb{R}^{n}\right)$. We call $(A, S)$ a SAP for the Weyl transform $W$ if there exists a positive number $C=C(A, S)$ such that

$$
\|g\|_{2}^{2} \leq C\left(\|g\|_{L^{2}\left(A^{c}\right)}+\left\|P_{S}^{\perp} W(g)\right\|_{H S}^{2}\right)
$$

for every $g \in L^{2}\left(\mathbb{C}^{n}\right)$.

In a similar way as to the Euclidean case, it can be shown that if $A$ has finite measure and the dimension of $S$ is finite, then $(A, S)$ is a SAP for $W$.

Remark 6.10. (a) Let $G$ be a step two nilpotent Lie group (without MW condition). Then $G$, as a set, can be realized by $\mathbb{R}^{2 n} \times \mathbb{R}^{r} \times \mathbb{R}^{k}$. Let $\Lambda$ be the set of all irreducible unitary representation of $G$ those participate in the Plancherel formula. Then $\Lambda$ can be identified with $\mathbb{R}^{k} \times \mathbb{R}^{r}$. For unexplained details about step two nilpotent Lie group, we refer to [11, 28, 29, 32.

A similar proof as to the Heisenberg group leads to the following analogue of Benedicks-Amrein-Berthier theorem on $G$.

Statement: Let $A \subset \mathbb{R}^{2 n}$ be a set of finite Lebesgue measure. Suppose $f \in$ $L^{1}(G)$ and $\{(x, y, t) \in G: f(x, y, t) \neq 0\} \subseteq A \times \mathbb{R}^{r} \times \mathbb{R}^{k}$. If $\hat{f}(\lambda, \mu)$ is a finite rank operator for each $(\lambda, \mu) \in \Lambda$, then $f=0$.

(b) Let $\mathcal{E}$ be a set of finite measure in $G$. If $\{(x, y, t) \in G: f(x, y, t) \neq 0\} \subseteq \mathcal{E}$ for some $f \in L^{1}(G)$, then it is natural to ask, whether there exists a nonzero function $f \in L^{1}(G)$ such that $\hat{f}(\lambda, \mu)$ has finite rank for every choice of $\lambda, \mu \in$ 
$\Lambda$. Since $G \cong \mathbb{R}^{2 n+r+k}$, if the projection of $\mathcal{E}$ into $\mathbb{R}^{2 n}$ has finite measure, then by Remark 6.10 (a) we get $f=0$. However, the other case is still open.

Acknowledgements: The authors wish to thank E.K. Narayanan and Rama Rawat for some fruitful suggestion and remarks. The first author gratefully acknowledges the support provided by IIT Guwahati, Government of India.

\section{REFERENCES}

[1] M. L. Agranovsky, V. V. Volchkov and L. A. Zalcman, Conical uniqueness sets for the spherical Radon transform, Bull. London Math. Soc. 31 (1999), no. 2, 231-236.

[2] W. O. Amrein and A. M. Berthier, On support properties of $L^{p}$-functions and their Fourier transforms, J. Functional Analysis 24 (1977), no. 3, 258-267.

[3] G. E. Andrews, R. Askey and R. Roy, Special Functions, Cambridge University Press, Cambridge, 1999.

[4] D. H. Armitage, Cones on which entire harmonic functions can vanish, Proc. Roy. Irish Acad. Sect. A 92 (1992), no. 1, 107-110.

[5] D. B. Babot, Heisenberg uniqueness pairs in the plane. Three parallel lines, Proc. Amer. Math. Soc. 141 (2013), no. 11, 3899-3904.

[6] M. Benedicks, On Fourier transforms of functions supported on sets of finite Lebesgue measure, J. Math. Anal. Appl. 106 (1985), no. 1, 180-183.

[7] A. Bonami and B. Demange, A survey on uncertainty principles related to quadratic forms, Collect. Math. 2006, Vol. Extra, 1-36.

[8] F. Canto-Martín, H. Hedenmalm and A. Montes-Rodríguez, Perron-Frobenius operators and the Klein-Gordon equation, J. Eur. Math. Soc. (JEMS) 16 (2014), no. 1, 31-66.

[9] A. Chattopadhyay, S. Ghosh, D. K. Giri and R. K. Srivastava, Heisenberg uniqueness pairs on the Euclidean spaces and the motion group, C. R. Math. Acad. Sci. Paris 358 (2020), no. 3, 365-377.

[10] A. Chattopadhyay, D. K. Giri and R. K. Srivastava, Uniqueness of the Fourier transform on certain Lie groups, arXiv:1607.03832,

[11] L. J. Corwin and F.P. Greenleaf, Representations of nilpotent Lie groups and their applications, Cambridge Studies in Advanced Mathematics, 18, Cambridge University Press, Cambridge, 1990.

[12] C. F. Dunkl, Boundary value problems for harmonic functions on the Heisenberg group, Canad. J. Math. 38 (1986), no. 2, 478-512.

[13] A. Fernández-Bertolin, K. Gröchenig and P. Jaming, From Heisenberg uniqueness pairs to properties of the Helmholtz and Laplace equations, J. Math. Anal. Appl. 469 (2019), no. 1, 202-219.

[14] M. Filaseta and T.-Y. Lam, On the irreducibility of the generalized Laguerre polynomials, Acta Arit. 105 (2002), 177-182.

[15] G. B. Folland and A. Sitaram, The uncertainty principle: a mathematical survey, J. Fourier Anal. Appl. 3 (1997), 207-238.

[16] D. K. Giri and R. Rawat, Heisenberg uniqueness pairs for the hyperbola, Bull. Lond. Math. Soc., doi: 10.1112/blms.12391 (to appear).

[17] D. K. Giri and R. K. Srivastava, Heisenberg uniqueness pairs for some algebraic curves in the plane, Adv. Math. 310 (2017), 993-1016.

[18] P. C. Greiner, Spherical harmonics on the Heisenberg group, Canad. Math. Bull. 23 (1980), no. 4, 383-396. 
[19] K. Gröchenig and P. Jaming, The Cramér-Wold theorem on quadratic surfaces and Heisenberg uniqueness pairs, J. Inst. Math. Jussieu 19 (2020), no. 1, 117-135.

[20] V. Havin and B. Jöricke, The Uncertainty Principle in Harmonic Analysis, Ergebnisse der Mathematik und ihrer Grenzgebiete (3) [Results in Mathematics and Related Areas (3)], 28, Springer-Verlag, Berlin, 1994.

[21] H. Hedenmalm and A. Montes-Rodríguez, Heisenberg uniqueness pairs and the KleinGordon equation, Ann. of Math. (2) 173 (2011), no. 3, 1507-1527.

[22] H. Hedenmalm and A. Montes-Rodríguez, The Klein-Gordon equation, the Hilbert transform, and dynamics of Gauss-type maps, J. Eur. Math. Soc. (JEMS) 22 (2020), no. $6,1703-1757$.

[23] H. Hedenmalm and A. Montes-Rodríguez, The Klein-Gordon equation, the Hilbert transform, and Gauss-type maps: $H^{\infty}$ approximation, J. Anal. Math. (2020) (to appear).

[24] A. J. Hogan, A qualitative uncertainty principle for unimodular groups of type I, Trans. Amer. Math. Soc. 340 (1993), no. 2, 587-594.

[25] P. Jaming and K. Kellay, A dynamical system approach to Heisenberg uniqueness pairs, J. Anal. Math. 134 (2018), no. 1, 273-301.

[26] N. Lev, Uniqueness theorem for Fourier transform, Bull. Sci. Math. 135 (2011), no. 2, 134-140.

[27] E. K. Narayanan and P. K. Ratnakumar, Benedicks' theorem for the Heisenberg group, Proc. Amer. Math. Soc. 138 (2010), no. 6, 2135-2140.

[28] S. Parui and R. P. Sarkar, Beurling's theorem and $L^{p}-L^{q}$ Morgan's theorem for step two nilpotent Lie groups, Publ. Res. Inst. Math. Sci. 44 (2008), no. 4, 1027-1056.

[29] S. Parui and S. Thangavelu, On theorems of Beurling and Hardy for certain step two nilpotent groups, Integral Transforms Spec. Funct. 20 (2009), no. 1-2, 127-145.

[30] V. Pati and A. Sitaram, Some questions on integral geometry on Riemannian manifolds, Ergodic theory and harmonic analysis (Mumbai, 1999), Sankhya Ser. A 62 (2000), no. 3, 419-424.

[31] F. J. Price and A. Sitaram, Functions and their Fourier transforms with supports of finite measure for certain locally compact groups, J. Funct. Anal. 79 (1988), no. 1, 166-182.

[32] S. K. Ray, Uncertainty principles on two step nilpotent Lie groups, Proc. Indian Acad. Sci. Math. Sci. 111 (2001), no. 3, 293-318.

[33] W. Rudin, Function theory in the unit ball of $\mathbb{C}^{n}$, Springer-Verlag, New York-Berlin, 1980.

[34] A. Sitaram, M. Sundari and S. Thangavelu, Uncertainty principles on certain Lie groups, Proc. Indian Acad. Sci. Math. Sci. 105 (1995), no. 2, 135-151.

[35] P. Sjölin, Heisenberg uniqueness pairs and a theorem of Beurling and Malliavin, Bull. Sci. Math. 135 (2011), 125-133.

[36] P. Sjölin, Heisenberg uniqueness pairs for the parabola, J. Fourier Anal. Appl. 19 (2013), no. 2, 410-416.

[37] C. D. Sogge, Oscillatory integrals and spherical harmonics, Duke Math. J. 53 (1986), no. $1,43-65$.

[38] R. K. Srivastava, Real analytic expansion of spectral projections and extension of the Hecke-Bochner identity, Israel J. Math. 200 (2014), no. 1, 171-192.

[39] R. K. Srivastava, Non-harmonic cones are sets of injectivity for the twisted spherical means on $\mathbb{C}^{n}$, Trans. Amer. Math. Soc. 368 (2016), no. 3, 1941-1957.

[40] R. K. Srivastava, Non-harmonic cones are Heisenberg uniqueness pairs for the Fourier transform on $\mathbb{R}^{n}$, J. Fourier Anal. Appl. 24 (2018), no. 6, 1425-1437.

[41] E. M. Stein and G. Weiss, Introduction to Fourier analysis on Euclidean spaces, Princeton Mathematical Series, No. 32. Princeton University Press (1971). 
[42] S. Thangavelu, Harmonic analysis on the Heisenberg group, Prog. Math., 159, Birkhuser, Boston, 1998.

[43] S. Thangavelu, An introduction to the uncertainty principle, Prog. Math., 217, Birkhauser, Boston, 2004.

[44] M. K. Vemuri, Benedicks theorem for the Weyl transform, J. Math. Anal. Appl. 452 (2017), no. 1, 209-217.

[45] F. J. G. Vieli, A uniqueness result for the Fourier transform of measures on the sphere, Bull. Aust. Math. Soc. 86 (2012), no. 1, 78-82.

[46] F. J. G. Vieli, A uniqueness result for the Fourier transform of measures on the paraboloid, Matematicki Vesnik 67 (2015), no. 1, 52-55.

Department of Mathematics, Indian Institute of Technology, Guwahati, INDIA 781039.

E-mail address: gsomnath@iitg.ac.in, rksri@iitg.ac.in 